\title{
Força de membros superiores e inferiores de idosas praticantes e não praticantes de ginástica funcional
}

Sálvio Santos de Souza Júnior ${ }^{1}$, Adriana Coutinho de Azevedo Guimarães², Simone Korn ${ }^{3}$, Leonessa Boing ${ }^{1}$, Zenite Machado²

\begin{abstract}
RESUMO
Objetivo: Buscou-se comparar nível de força de membros superiores e inferiores de idosas praticantes e não praticantes de ginástica funcional, Florianópolis, SC. Metodologia: Trata-se de um estudo quase-experimental, com amostra constituída por 101 idosas, sendo 50 participantes de atividades de ginástica funcional e 51 não participantes de nenhum programa sistemático de atividade física. Ambos os grupos passaram pelos testes da Bateria de Rikli e Jones (1999), avaliação de força e resistência de membros superiores (flexão do antebraço) e inferiores (levantar e sentar na cadeira), realizadas em agosto (pré-teste) e dezembro (pós-teste) de 2012. Fez-se uso da estatística descritiva e do teste "t" de Student, $p<0,05$. Resultados: Evidenciam que o grupo de praticantes de ginástica funcional apresentam força de membros superiores e inferiores em melhores níveis. Conclusão: A participação em um programa regular e orientado de ginástica funcional contribui para a prevenção e minimização do declínio da força em idosas.
\end{abstract}

Descritores: Envelhecimento; Força Muscular; Treinamento.

\section{Strength of lower and upper limbs of elderly practicing and not practicing functional exercise}

\begin{abstract}
Objectives: We aimed to compare level of strength of upper and lower limbs of elderly practitioners and non-practitioners of functional fitness, Florianópolis, SC. Methodology: A quasi-experimental study with a sample of 101 elderly, 50 participants from 51 functional fitness activities and not participating in any systematic program of physical activity. Both groups went through the battery Rikli and Jones (1999), evaluation of strength and endurance of upper limb (forearm flexion) and lower (up and sit in the chair), occurred in July (pre-test) and December (posttest) 2012. We made use of the descriptive statistics and the " $t$ " test, $p<0.05$. Results: Indicate that the group of practitioners has functional fitness strength of upper and lower levels at best. Conclusion: The findings suggest that participation in a regular program of exercise and targeted functional fitness, contributes effectively to the prevention and minimization of the decline in strength in elderly women.
\end{abstract}

Descriptors: Aging; Muscle Strength; Training.

${ }^{1}$ Graduado do Curso de Educação Física pela Universidade do Estado de Santa Catarina (UDESC), Florianópolis, SC, Brasil.

${ }^{2}$ Doutora em Motricidade Humana pela Faculdade de Motricidade Humana (FMH) da Universidade Técnica de Lisboa (UTL), Lisboa, POR, Portugal.

${ }^{3}$ Fisioterapeuta pela Universidade do Sul de Santa Catarina (UNISUL), Florianópolis, SC, Brasil. 


\section{Introdução}

O aumento da população de idosos trata-se, atualmente, de uma realidade demográfica cada vez mais presente na população mundial ${ }^{1,2}$. O mundo está em processo de envelhecimento ${ }^{2}$, e no Brasil, este vem ocorrendo de forma acelerada nas últimas décadas ${ }^{3,4}$. Estima-se para 0 ano de 2050, no Brasil, bem como em todo o mundo, a existência de mais idosos que crianças abaixo de 15 anos $^{2}$, e segundo a Organização Mundial da Saúde 5 já em 2025, o Brasil será 0 sexto país do mundo em número de idosos.

Segundo alguns autores, o envelhecimento pode ser definido como uma série de processos que ocorrem nos organismos vivos e com o passar dos anos leva a alterações funcionais e a uma perda da adaptabilidade ${ }^{3}$. É um processo fisiológico que não necessariamente ocorre paralelamente à idade cronológica e que apresenta considerável variação individual ${ }^{6}$. Doenças crônicas e degenerativas aparecem com o decorrer do envelhecimento, contribuindo para a diminuição das habilidades necessárias para a vida diária e um importante marcador de um envelhecimento bem sucedido e de uma ótima qualidade de vida nos idosos é a capacidade funcional ${ }^{2,4,7}$.

Para uma efetiva prevenção, ou mesmo para a redução das perdas funcionais que estão associadas ao envelhecimento, sugere-se a inclusão num programa regular de exercícios físicos ${ }^{4,8,9}$. 0 declínio da performance física pode ser minimizado por meio dessa prática, o que tem sido relacionado a melhoras significativas em aspectos psicológicos e físicos do idoso praticante, auxiliando positivamente na função corporal, mantendo a capacidade física, melhorando a qualidade de vida e diminuindo o risco de quedas ${ }^{8,10}$.

O treinamento sistemático com pesos em idosos pode promover aumento da força, da massa muscular e da flexibilidade ${ }^{11,12}$. Se apenas uma forma de exercício tiver que ser escolhida para promover a melhora na capacidade funcional de idosos, sugerese $o$ treinamento com pesos, pois as principais atividades cotidianas, presentes na vida do idoso, envolvem capacidades que são aprimoradas durante a prática do treinamento com pesos ${ }^{13}$. A ginástica funcional, por meio do treinamento de força, exercícios aeróbios, de flexibilidade e de equilíbrio14, caracteriza-se por objetivar a melhora das capacidades funcionais para transferência dos benefícios e adaptações da vida cotidiana ou para determinado gesto esportivo ${ }^{15}$.

O treinamento de força, por meio da ginástica funcional, é recomendado para idosos, tendo como objetivo o aumento da força e potência muscular, visto que se observa uma melhora nas capacidades funcionais ${ }^{16}$. Ainda, pode ser vista como uma possibilidade terapêutica não-farmacológica na manutenção da pressão arterial e da frequência cardíaca e de modificação do estilo de vida ${ }^{15}$.

No entanto a literatura específica não apresenta ainda uma produção substancial e conclusiva a respeito dos ganhos de força em idosos por meio de um programa de ginástica funcional. Sendo assim, delineou-se um estudo com o objetivo de comparar o nível de força entre idosas, mulheres, praticantes e não praticantes de ginástica funcional.

\section{Metodologia}

O estudo caracteriza-se como quase-experimental, mais especificamente com design do grupo de controle nãoequivalente. Esse recurso investigativo é idêntico, em todos os aspectos ao design experimental, exceto no que diz respeito ao processo de seleção aleatória dos sujeitos ${ }^{17}$. Para reduzir a desvantagem da não utilização de grupos amostrais intactos ou randomizados, este modelo prevê a participação concomitante de um grupo sujeito a um tratamento (experimental) e um grupo de controle, ambos submetidos a um pré e pós-teste. O esquema do delineamento foi o que segue:

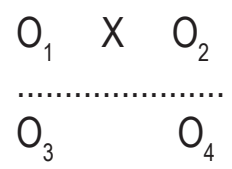

A pesquisa foi submetida ao Comitê de Ética em Pesquisa em Seres Humanos da Universidade do Estado de Santa Catarina e aprovada sob o protocolo № 63431 , em 30/07/2012, e foi conduzida de acordo com os padrões éticos exigidos. A amostra foi constituída inicialmente por um grupo de 54 idosas que participam das atividades de ginástica funcional oferecida pelo Programa "Viver Ativo" da Prefeitura do Município de Florianópolis e outro grupo de 58 idosas que não participavam de nenhum programa sistemático de atividade física. A constituição da amostra obedeceu aos seguintes critérios de inclusão: ter idade igual ou superior a 60 anos e aceitação por meio da assinatura do termo de consentimento livre e esclarecido. 
O instrumento utilizado para a coleta de dados foi o teste desenvolvido por Rikli e Jones ${ }^{18}$. O referido protocolo avalia as seguintes variáveis: "força e resistência dos membros inferiores e superiores", "flexibilidade dos membros inferiores e superiores", "velocidade, agilidade e equilíbrio dinâmico" e "resistência aeróbia". Entretanto, a variável selecionada para o estudo foi a "força e resistência dos membros inferiores e superiores". Os testes que avaliaram estas valências foram respectivamente o levantar e sentar na cadeira e o de flexão de antebraço.

Inicialmente entrou-se em contato com a Secretaria Municipal de Assistência Social de Florianópolis a fim de localizar os possíveis sujeitos da amostra. Estes foram identificados entre os indivíduos que participavam de grupos de convivência, para constituirem o grupo controle, e entre aqueles que participavam de grupos de ginástica, para formar o grupo que seria submetido à intervenção. Ambos os grupos foram integrados por participantes do programa "Viver Ativo", o grupo experimental foi formado pelos idosos que faziam parte da modalidade de ginástica funcional, que é oferecida pelo "Viver Ativo" a 26 grupos de idosos, cuja periodicidade é de três sessões semanais, com duração de aproximadamente uma hora cada sessão, divididas em três partes: inicial (aquecimentos e alongamentos), principal (exercícios de fortalecimento muscular, aeróbios, de flexibilidade, de equilíbrio, entre outros) e final (relaxamento). 0 grupo controle foi também formado pelos participantes do programa "Viver Ativo", no entanto, por aqueles que participavam apenas dos grupos de convivência, e que não realizavam a prática de atividade física.

A coleta de dados de ambos os grupos foi realizada em duas etapas. Na primeira etapa, realizada na primeira quinzena de agosto 2012, ambos os grupos foram submetidos a uma avaliação (pré-teste). 0 instrumento utilizado nas avaliações foi o teste desenvolvido por Rikli e Jones ${ }^{18}$. Os testes que avaliaram as valências selecionadas foram respectivamente o levantar e sentar na cadeira e o de flexão de antebraço, onde os sujeitos foram encorajados a repetir, o maior número de vezes possível, o movimento correto com o tempo de 30 segundos de duração. Depois desta etapa o grupo experimental participou do programa de ginástica funcional durante um período de cinco meses, enquanto o grupo controle permaneceu sem realizar atividade física sistemática pelo mesmo período correspondente ao outro grupo. As atividades desenvolvidas pelo grupo experimental constaram de exercícios aeróbios, de fortalecimento muscular e de flexibilidade, com frequência de três sessões semanais e duração de 60 minutos por sessão. Os participantes do estudo obtiveram uma frequência igual ou superior a $75 \%$. Após cinco meses, ambos os grupos passaram por uma segunda avaliação (pós-teste), caracterizando assim, a segunda etapa do estudo. Todos os testes foram repetidos nas mesmas condições em todos os períodos de coleta. A exclusão das idosas aconteceu apenas pelas faltas ocorridas durante 0 processo de coleta, especificamente na segunda etapa do estudo, onde foram excluídas quatro idosas do grupo de praticantes e sete idosas do grupo de não praticantes, por não comparecerem à segunda avaliação.

Além da utilização dos valores médios obtidos em cada uma das variáveis, o protocolo permite a categorização dos resultados em três distintos grupos: abaixo da média, quando apresenta valor inferior ao percentil 50, na média, quando apresenta valores correspondentes ao percentil 50 e acima da média, quando os valores ultrapassam o percentil 50. Convém salientar que a classificação respeita escalões etários.

Para garantir a confiabilidade dos resultados procedeu-se a verificação de possível diferença entre as médias de idade dos grupos de praticantes com não praticantes, haja vista ser a idade, além do sexo, um dos fatores que influencia o declínio da força. Sendo assim, a inexistência de diferença significativa entre as médias de idade dos dois grupos (Tabela 1) confere maior consistência aos resultados.

Tabela 1 - Média de idade dos grupos de praticantes e não praticantes de Ginástica Funcional.

\begin{tabular}{c|c|c|c|c}
\hline Grupo & N & Média & DP & Valor de $\mathbf{p}$ \\
\cline { 1 - 4 } Praticantes & 50 & 69,80 & 6,51 & \multirow{2}{*}{$0,713^{*}$} \\
\hline Não praticantes & 51 & 70,27 & 6,39 & \\
\hline
\end{tabular}

*Resultado obtido por meio do teste "t" Student para amostras independentes; n: número de participantes; Média: média de idade; DP: desvio padrão

Para traçar o perfil da amostra foi confeccionada uma planilha eletrônica no programa Excel, utilizando a estatística descritiva (média e desvio padrão). Após, os dados foram transportados para o programa SPSS (Statistical Package for Social Sciences) 17.0. Para análise das diferenças entre as médias de idade e entre os resultados dos testes dos dois grupos utilizou-se o teste "t" de Student para amostras independentes e para as comparações intragrupos (pré e pósteste) foi utilizado o teste "t" de Student pareado, ambos com nível de significância de $p<0,05$. 


\section{Resultados}

Os resultados são apresentados inicialmente em relação às médias obtidas por cada grupo, nas variáveis investigadas (Tabela 2) e, posteriormente, por meio das frequências e percentuais verificados em cada uma das categorias de classificação do protocolo (Tabela 3). A Tabela 2 permite a visualização de comparações de duas ordens.

No sentido vertical possibilita a comparação entre o pré e o pós-teste intragrupos e no horizontal, a comparação intergrupos em cada uma das variáveis e testagens.

Tabela 2 - Valores médios do número de repetições dos grupos praticantes e não praticantes de ginástica funcional

\begin{tabular}{c|c|c|c|c|c}
\hline Variáveis & \multicolumn{2}{|c|}{ Praticantes } & \multicolumn{2}{c|}{ Não praticantes } & \multirow{2}{*}{ Valor de $\mathbf{p}^{*}$} \\
\cline { 1 - 5 } & Média & DP & Média & DP & \\
\hline MS1 & 18,38 & 3,16 & 14,35 & 2,76 & $<0,001$ \\
\hline MS2 & 19,50 & 3,28 & 13,78 & 2,52 & $<0,001$ \\
\hline Valor de $p$ & \multicolumn{2}{|c|}{$<0,001$} & \multicolumn{2}{|c|}{0,005} & \\
\hline Ml1 & 17,28 & 3,53 & 13,59 & 2,76 & $<0,001$ \\
\hline Ml2 & 16,08 & 3,96 & 13,71 & 2,92 & 0,001 \\
\hline Valor de $p$ & \multicolumn{3}{|c|}{$<0,001$} & \multicolumn{3}{c}{0,703} & \\
\hline
\end{tabular}

"Resultado obtido por meio do teste "t" de Student para amostras independentes; MS1: membros superiores pré teste; MS2: membros superiores pós teste; MI1: membros inferiores pré teste; MI2: membros inferiores pós teste.

No sentido vertical, na primeira avaliação do teste de flexão de antebraço, o grupo de praticantes apresentou um valor médio de 18,38 flexões, e após cinco meses, quando as idosas foram submetidas ao pós teste o valor médio de repetições subiu para 19,50, enquanto que o grupo controle obteve 14,35 repetições no pré-teste, declinando para 13,78 no pós-teste. Já no teste de levantar e sentar da cadeira, que avaliou a força de membros inferiores, o grupo de praticantes obteve o número de 17,28 repetições na primeira avaliação, e na segunda passou para 16,08. Já o grupo controle passou de 13,59 no pré-teste, para 13,71 no pós-teste. Como pode ser verificado, entre o grupo de praticantes verificou-se aumento significativo da força de membros superiores, e no grupo de não praticantes, ocorreu redução significativa na força dos membros superiores, embora tenha havido uma ligeira melhoria nas médias de força dos membros inferiores, esse aumento não foi significativo. No sentido horizontal, quando se analisa o resultado entre os grupos fica evidente que o nível de força do grupo de praticantes é significativamente superior nas duas variáveis, tanto no pré como no pós-teste.

Tabela 3 - Categorização dos grupos praticantes (P1) e não praticantes (P2) de ginástica funcional na força de membros superiores e inferiores por meio dos critérios do protocolo de Rikli e Jones ${ }^{19}$.

\begin{tabular}{c|c|c|c|c|c|c|c|c}
\hline \multirow{2}{*}{ Variável } & \multirow{2}{*}{ Grupo } & \multicolumn{2}{|c|}{ Abaixo da média } & \multicolumn{2}{|c|}{ Normal } & \multicolumn{2}{c|}{ Acima da média } & \multirow{2}{*}{ Valor de $\mathrm{p}$} \\
\cline { 3 - 8 } & & $\mathrm{N}$ & $\%$ & $\mathrm{~N}$ & $\%$ & $\mathrm{~N}$ & $\%$ & \\
\hline \multirow{2}{*}{ MS1 } & $\mathrm{P} 1$ & 2 & 4,0 & 3 & 6,0 & 45 & 90,0 & \multirow{2}{*}{$<0,001$} \\
\cline { 2 - 8 } & $\mathrm{P} 2$ & 24 & 47,1 & 5 & 9,8 & 22 & 43,1 & \\
\hline \multirow{2}{*}{ MS2 } & $\mathrm{P} 1$ & 6 & 12,0 & 9 & 18,0 & 35 & 70,0 & \multirow{2}{*}{0,021} \\
\cline { 2 - 8 } & $\mathrm{P} 2$ & 18 & 35,3 & 8 & 15,7 & 25 & 49,0 & \\
\hline \multirow{2}{*}{ MI1 } & $\mathrm{P} 1$ & - & - & 2 & 4,0 & 48 & 96,0 & \multirow{2}{*}{$<0,001$} \\
\cline { 2 - 8 } & $\mathrm{P} 2$ & 27 & 53,0 & 12 & 23,5 & 12 & 23,5 & \\
\hline \multirow{2}{*}{ MI2 } & $\mathrm{P} 1$ & 2 & 4,0 & 4 & 8,0 & 44 & 88,0 & \multirow{2}{*}{$<0,001$} \\
\cline { 2 - 7 } & $\mathrm{P} 2$ & 20 & 39,2 & 7 & 13,7 & 24 & 47,1 & \\
\hline
\end{tabular}

Na tabela 3 pode-se verificar que o primeiro teste que avaliou a força e resistência dos membros superiores identificou que $90 \%$ das idosas do grupo de praticantes estão acima da média para essa população, conforme a classificação de Rikli e Jones ${ }^{19}$, enquanto que no grupo controle, um pouco menos da metade, ou seja, $43,1 \%$ delas estão acima da média. No pós-teste, que avaliou a mesma variável, o número de praticantes acima da média caiu para $70 \%$, e 0 grupo controle subiu para $49 \%$. A existência de diferença significativa entre os dois grupos, nas duas avaliações, evidencia que desempenho de força de membros superiores entre as praticantes é superior quando comparado com não praticantes. 
O mesmo ocorreu no teste de levantar e sentar da cadeira, onde na primeira avaliação o grupo de praticantes atingiu $96 \%$ acima da média, contra $23,5 \%$ do grupo de não praticantes. No pós-teste, $88 \%$ no grupo praticante continuaram acima da média, e houve uma melhora no grupo de não praticantes. Mesmo assim o grupo de praticantes leva uma vantagem estatisticamente significativa em relação ao grupo não praticante.

\section{Discussão}

Os resultados apontam, diante da comparação entre os níveis de força de membros superiores e inferiores de idosas praticantes e não praticantes de ginástica funcional, que apesar de alguns declínios de um teste para outro (pré e pós-teste), o desempenho das idosas praticantes apresentou-se como superior quando comparado aos resultados das não praticantes.

$O$ efeito que os exercícios físicos têm sobre o equilíbrio, a força e o tempo de reação em idosos tem mostrado melhoras significativas na força e no equilíbrio corporal após um programa de atividade física, em comparação com aquele idoso que não se exercita, evidenciando assim, que a prática de atividade física é de grande importância para a promoção da saúde nessa etapa da vida ${ }^{10}$. Um treinamento de força realizado com 1.132 indivíduos de ambos os sexos, com idades entre $21 \mathrm{e}$ 80 anos, participantes de um programa de atividade física, mostrou que as faixas etárias entre 21 a 40 anos e 41 a 60 anos aumentaram em média 1,04kg de músculo, enquanto os indivíduos de 61 a 80 anos aumentaram em media 1,09kg ${ }^{19}$.

Conforme os resultados apresentados na tabela 2, no sentido vertical, em relação aos níveis de força de membros superiores intragrupos, verifica-se uma melhora estatisticamente significativa $(p<0,001)$ no desempenho do grupo praticante em relação ao pré e pós-teste. Esses resultados são semelhantes aos de outro estudo, que verificou um ganho de força de até $227 \%$ em idosos, após um programa de treinamento de força muscular, com duração de 12 semanas $^{20}$. Corroborando com outros resultados, que apontam ganhos significativos de força máxima em idosas que realizaram um treinamento de força, durante três meses de intervenção, com frequência de três vezes por semana ${ }^{21}$. Ainda, semelhantemente com estudo que comparou três programas de atividade física (Hidroginástica, Caminhada e Lian Gong), a partir da capacidade funcional, e foi realizado em 113 idosas, e mostrou que o grupo de hidroginástica, no teste que avaliou a força dos membros superiores, foi superior aos demais grupos, com uma diferença significativa de $p<0,001^{22}$. Já outro estudo comparativo analisou, dentre outras valências, a força muscular de idosas praticantes de ginástica e de hidroginástica. Concluiu que não houve diferença significativa na força muscular dos membros inferiores entre os dois grupos investigados, porém foram superiores na média de referência para a população geral23.

Ainda na tabela 2 identifica-se que o grupo sedentário obteve um declínio em relação à força de membros superiores entre o pré e pós-teste. Tal declínio foi estatisticamente significativo $(p=0,005)$. Com o passar dos anos, ocorre uma queda do desempenho nas capacidades funcionais, como a perda de flexibilidade, agilidade, força e resistência, devido à redução do número e tamanho das fibras musculares ${ }^{24}$. Em termos percentuais, a partir dos cinquenta anos a taxa de declínio é de 12 a $15 \%$ por década ${ }^{13}$. Um treinamento com pesos, planejados e adequados pode resultar em aumentos significativos na força, na densidade mineral óssea e na flexibilidade ${ }^{25}$. Um estudo realizado com idosos submetidos a um treinamento resistido de força, pelo período de 12 semanas, apontou melhoras na flexibilidade ${ }^{15}$. Outro estudo sobre os efeitos de três modalidades de atividade física na capacidade funcional dos idosos, concluiu que idosos previamente treinados por quatro anos em média, que participaram de um protocolo de treinamento de atividade física geral, obtiveram melhoras significativas nos componentes agilidade e equilíbrio dinâmico, e resistência de força ${ }^{26}$.

Com relação à força de membros inferiores verifica-se que o grupo de praticantes declinou a sua média de repetições. Existe a hipótese de que as idosas, de uma forma em geral, utilizam mais os membros superiores do que os inferiores nas atividades da vida diária, pois passam mais tempo sentadas, seja bordando, tricotando, costurando, ou realizando outras atividades que utilizam os membros superiores, e em conjunto com a atividade física, acabam retardando de forma mais rápida a perda dessa musculatura. Em estudo que teve como objetivo verificar o declínio na força de membros inferiores e superiores e na agilidade em mulheres praticantes de atividade física, identificaram que há uma maior perda da força de membros inferiores em relação aos superiores ${ }^{27}$. Os autores justificam essa diferença justamente em razão de uma menor utilização dessa musculatura com o passar dos anos, uma vez que os idosos permanecem sentados na maior parte do tempo, não havendo locomoção. Explicam, portanto, que em membros superiores esta mudança não ocorre com essa magnitude, pois as atividades diárias que se utilizam dessa musculatura não sofrem tantas modificações. Outra possível explicação é o fenômeno natural do envelhecimento, conhecido como sarcopenia, que se associa ao declínio progressivo 
da massa e, consequentemente, da função muscular (força, potência e resistência) dos idosos ${ }^{28}$. E esse processo continua ocorrendo naqueles indivíduos que treinam, sendo relatado apenas uma desaceleração ou minimização deste processo ${ }^{29}$.

Ainda na tabela 2, no sentido horizontal, como era o esperado, verifica-se que o grupo de praticantes obteve resultados superiores aos do grupo de não praticantes. Nas duas variáveis houve uma diferença estatisticamente significativa com valores de "p" menores que 0,001 tanto no pré, como no pós-teste. O resultado evidencia que idosos fisicamente ativos, em relação à força, têm melhores desempenhos, se comparados com idosos sedentários. Em um estudo investigando a hidroginástica, realizado com 74 idosas (37 praticantes e 37 não praticantes), com objetivo de verificar o efeito da prática de hidroginástica sobre a aptidão física do idoso foi observado um melhor desempenho no final da intervenção do grupo praticante em relação ao não praticante, tanto no teste que avaliou a força de membros superiores quanto de membros inferiores, havendo assim uma diferença significativa $(p<0,001)^{30}$, similar aos resultados apresentados na tabela 2, no entanto, trata-se de uma modalidade que em paralelo trabalha o equilíbrio, o que pode impedir comparações entre os resultados. Em contrapartida, estudo realizado na Universidade Regional do Noroeste do Rio Grande do Sul, que objetivou comparar o nível de aptidão física de dez idosas fisicamente ativas, participantes das atividades no "Grupo de Atividade Física Boa Idade", e de outras dez que não participam de nenhum programa sistêmico de atividade física, concluiu-se, em relação a força muscular, que o desempenho das idosas praticantes também foi superior ao grupo sedentário, apontando-se novamente uma diferença significativa $(p=0,02)$ entre os dois grupos ${ }^{31}$, o que corrobora com os achados do presente estudo.

De forma semelhante, num estudo que avaliou entre outras variáveis, a força de membros superiores e inferiores, em 20 idosas praticantes e 20 não praticantes de atividades físicas, na região do Vale dos Sinos, RS, concluíram que as idosas praticantes de atividades físicas (alongamentos, ginástica, dança, recreação e corrida orientada) do Programa de Ginástica para a Terceira idade de Sapiranga, obtiveram um desempenho superior significativo tanto no teste que avaliou a força dos membros superiores quanto inferiores $(p<0,001)^{32}$. Outros autores, ao compararem a força de membros inferiores entre idosas praticantes e não praticantes de ginástica recreativa, também obtiveram resultados positivos a favor do grupo de praticantes ${ }^{33}$, demonstrando que a literatura consolida os benefícios da prática de atividade física em relação às variáveis de força em idosas.

A Tabela 3 apresenta a categorização dos resultados em três grupos: abaixo da média (inferior a 50\%), normal (corresponde a 50\%), e acima da média (acima de 50\%), segundo a classificação de Rikli e Jones ${ }^{18}$. De uma forma geral, o grupo de praticantes teve o maior percentual de idosos que conseguiu ultrapassar os valores de referência considerados como normal, segundo o protocolo dos referidos autores. Em ambos os testes, tanto na primeira como na segunda avaliação, verifica-se uma diferença estatisticamente significativa entre praticantes e não praticantes, com valores de "p" que variam entre <0,001 e 0,021. Ao comparar os grupos "abaixo da média" e "acima da média" da referida tabela, fica evidente que o maior percentual de idosos que atingiu valores acima da média, é justamente o grupo de praticantes. Em concordância com esses resultados, outro estudo aponta que ao analisarem 1004 indivíduos praticantes de atividades físicas, com idades entre 50 a 86 anos, identificaram que as médias da amostra estudada foram mais altas do que os valores de referência ${ }^{34}$. Outros autores que tiveram como objetivo comparar a aptidão física entre idosos praticantes de tênis e sedentários do gênero masculino mostrou que os praticantes de tênis apresentaram um desempenho médio $38 \%$ maior na força e resistência de membros inferiores e 35\% maior na força de membros superiores, comparando com o grupo de idosos sedentários ${ }^{35}$. Embora tal estudo tenha sido feito apenas com indivíduos do sexo masculino, fica evidente que idosos fisicamente ativos respondem de forma superior o desempenho relacionado à capacidade funcional.

\section{Considerações Finais}

Os resultados evidenciam que o grupo de praticantes de ginástica funcional conseguiria atingir valores superiores nos testes que avaliaram tais forças, com diferenças estatisticamente significativas em comparação ao grupo de não praticantes, o que comprova que a participação desses idosos no programa de atividade física "Viver Ativo", oferecido pela Prefeitura Municipal de Florianópolis, é de grande importância para os mesmos e que tal participação pode ter uma influência direta nos resultados obtidos.

Conclui-se então que a participação em um programa regular e orientado de ginástica funcional, mesmo não apresentando uma especificidade do treinamento de força, contribui de forma efetiva para a prevenção e minimização do declínio da força em idosos. Os resultados do estudo mostraram que idosos fisicamente ativos respondem melhor aos trabalhos que necessitam da força muscular, quando comparados com idosos sedentários. 


\section{Referências Bibliográficas}

1. Carvalho J, Soares JMC. Envelhecimento e Força Muscular: breve revisão. Rev Port Cien Desp 2004;4(3):79-93.

2. Pilger $C$, Menon UM, Mathias TAF. Capacidade funcional de idosos atendidos em unidades básicas de saúde do SUS. Rev Bras Enferm. 2013; 66(6): 907-13.

3. Ministério da Saúde (Brasil). Envelhecimento e saúde da pessoa idosa. Caderno de Atenção Básica 2007;1(19):8.

4. Queiroz BM, Coqueiro RS, Neto JSL, Borgatto AF, Barbosa AR, Fernandes MH. Inatividade física em idosos não institucionalizados: estudo de base populacional. Ciência \& Saúde Coletiva, 2014;19(8):3489-3496.

5. Organização Mundial da Saúde (OMS), Organização Pan-Americana da Saúde (OPAS). Envelhecimento ativo: uma política de saúde. Brasília: OMS, OPAS; 2005.

6. Assumpção CO, Bartholomeu NJ, Pellegrinote IL, Montebelo MI. Controle da Intensidade Progressiva de Exercícios Localizados em Mulheres Idosas por Meio de Percepção Subjetiva de Esforço. Rev. Educ. Fís/UEM. 2008;19(1): 33-39.

7. Reis LA, Torres GV. Influência da dor crônica na capacidade funcional de idosos institucionalizados. Rev Bras Enferm. 2011;64(2):274-80.

8. Finnegan S, Bruce J, Lamb SE, Griffiths F. Predictors of attendance to group exercise: a cohort study of older adults in long-term care facilities. Geriatrics. 2015;15(37):1-12.

9. Kura, GG, Ribeiro LSP, Niquetti R, Tourinho Filho H. Nível de atividade física, IMC e índices de força muscular estática entre idosas praticantes de hidroginástica e ginástica. RBCEH 2004;1(2):30-40.

10. Ignasiak Z, Sławińska T, Dąbrowski A, Rowiński R. The structure of physical activity in seniors from lower Silesia. Rocz Panstw Zakl Hig. 2013;64(1):67-73.

11. American College of Sports Medicine: Position stand. Progression models in resistance training for healthy adults. Med Sci Sports Exerc 2002;34(2):364-380.

12. Carneiro N, Ribeiro AS, Nascimento MA, Gobbo LA, Schoenfeld BJ, Júnior AA, Gobbi S, Oliveira AR, Cyrino ES. Effects of different resistance training frequencies on flexibility in older women. Clinical Interventions in Aging 2015:10; 531-538.

13. Hunter GR, Mc Carthy JP, Bamman MM. Effects of resistance training on older adults. Sports Med 2004;34(5)329-348. 14. Misic MM, Valentine RR, Rosengren KS, Woods JA, Evans EM. Impact of Training Modality on Strength and Physical Function in Older Adults. Gerontology 2009;55:411-416.

15. Botelho LP, Vale RGS, Cader SA, Senna GW, Gomes MCV, EHM Dantas. Efeito da ginástica funcional sobre a pressão arterial, frequência cardíaca e duplo produto em mulheres. Acta Scientiarum. Health Sciences, 2011;33(2):119-125.

16. Barry BK, Carson RG. The Consequences of Resistance Trainning for Movement Control in Older Adults. J Gerontol: Med Sciences 2004;59(7):730-754.

17. Tuckman BW. Manual de investigação em educação. Lisboa: Fundação Calouste GulbenKian: 2000.

18. Rikli R, Jones CJ. Functional fitness normative scores for community-resing older adults. J Aging Phys Act. 2008.

19. Westcott W, Baechle T. Treinamento de força para a terceira idade. São Paulo: Manole, 2001.

20. Frontera WR, Meredith CN, O'Reilly KP, Evans WJ. Strength training and determinants of VO2 max in older man. J Appl Physiol 1990;68:329-333.

21. Geraldes AAR. Efeitos do treinamento contra resistência sobre a força muscular e o desempenho de habilidades funcionais selecionadas em mulheres. Rio de Janeiro. Dissertação [Mestrado em Ciência da Motricidade Humana]. Universidade Castelo Branco, 2002.

22. Nunes MES, Santos $S$. Avaliação funcional de idosos em três programas de atividade física: caminhada, hidroginástica e Lian Gong. Rev Port Cien Desp 2009;9(2-3):150-159.

23. Araujo RCM, Barbosa MTS. Análise comparativa da força muscular de mulheres idosas praticantes de ginástica e hidroginástica. Rev Dig Edu Fis, Ipatinga 2007;2(1). [acesso em: 15/08/2012]. Disponível em: http://www.unilestemg.br/ movimentum/Artigos_V2N1_em_pdf/movimentum_v2_n1_araujo_rita.pdf

24. Hughes VA, Frontera WR, Roubenoff R, Evans WJ, Singh MA. Longitudinal changes in body composition in older men and women: role of body weight change and physical activity. Am J Clin Nutr 2002;76:473-481.

25. Rabelo HT. Os efeitos do treinamento contra resistência no desempenho das atividades da vida diária de mulheres idosas. Brasília. Dissertação [Mestrado em Educação Física] Universidade Católica de Brasília, 2002.

26. Ueno DT, Gobbi S, Teixeira CVR, Sebastião E, Prado AKG, Costa JLR, Gobbi LTB, et al. Efeitos de três modalidades de atividade física na capacidade funcional de idosos. Rev Bras Edu Fis Esporte 2012;26(2). 
27. Andrade EL, Matsudo SM, Matsudo VKR. Performance neuromotora em mulheres ativas. Rev Bras Ativ Fis Saude 1995;1(2):5-14.

28. Pícoli TS, Figueiredo LL de, Patrizzi LJ. Sarcopenia e envelhecimento. Fisioter. mov. (Impr). 2011; 24(3):455-462.

29. Neto, LGWD. Os benefícios do treinamento de força no processo do envelhecimento: estudo de revisão de artigos científicos. Porto Alegre. Trabalho de Conclusão de Curso [Curso de Educação Física - Bacharelado]. Universidade Federal do Rio Grande de Sul, 2009.

30. Alves RV, Mota J, Costa MC, Alves JGB. Aptidão física relacionada à saúde de idosos: influência da hidroginástica. Rev Bras Med Esporte 2004;10(1):31-37.

31. Berlezi EM, Rosa PV, Souza ACA, Schneider RH. Comparação antropométrica e do nível de aptidão física de mulheres acima de 60 anos praticantes de atividade física e não praticantes. Rev Bras Geriatr Gerontol 2006;9(3): 49-66.

32. Fraccari VLK, Piccoli JCJ, Quevedo DM. Aptidão física relacionada à saúde de idosas da região do Vale dos Sinos, RS: um estudo ex post-facto. Rev Bras Geriatr Gerontol 2012;15(4):651-660.

33. Silva TCL, Costa EC, Guerra RO. Resistência aeróbica e força de membros inferiores de idosos praticantes e não praticantes de ginástica recreativa em um centro de convivência. Rev Bras Geriatr Gerontol 2011;14(3):535-542.

34. Carvalho RBCC, Madruga VA. Aptidão física relacionada à saúde em praticantes de atividades físicas de 50 a 86 anos. Rev Bras Ciênc Mov 2010;18(3):79-87.

35. Gripp F, Miranda AS, Bittencourt D, Gripp W, Lacerda ACR. Comparação da aptidão física entre idosos praticantes de tênis e sedentários do gênero masculino. Coleç. Pesqui. Educ. Fís. 2008;7(2):143-150.

\section{Sálvio Santos de Souza Júnior}

Endereço para correspondência - Rua Joaquim Palhares, $n^{\circ}$ 608, apto 1402 - Praça da Bandeira CEP: 20.260-085, Cidade: Rio de Janeiro, RJ, Brasil.

E-mail: ssjunior28@gmail.com

Lattes: http://lattes.cnpq.br/1046928210102996

Adriana Coutinho de Azevedo Guimarães - nanaguim@terra.com.br

Simone Korn - simonekorn@gmail.com

Leonessa Boing - leonessaboing@gmail.com

Zenite Machado - zenite13@yahoo.com.br

Enviado em 12 de outubro de 2014. Aceito em 06 de maio de 2015. 